\title{
Mössbauer Studies of Core-Shell Nanoparticles.
}

\author{
B. Kalska-Szostko ${ }^{a}$, M. Cydzik $^{a}$, D. SatuŁa ${ }^{b}$ And M. Giersig ${ }^{c}$ \\ ${ }^{a}$ Institute of Chemistry, University of Białystok, Hurtowa 1, 15-399 Białystok, Poland \\ ${ }^{b}$ Department of Physics, University of Białystok, Lipowa 41, 15-424 Białystok, Poland \\ ${ }^{c}$ Helmholtz - Centre Berlin, Glinker Strasse 100, Berlin, Germany
}

\begin{abstract}
The ferrite magnetic nanoparticles with core-shell structures were obtained in two step preparation process. The Mössbauer spectra obtained for particles of pure maghemite or magnetite and two layered core-shell one the magnetite on maghemite and maghemite on magnetite are very different from each other. The presented results show that interparticle and intraparticle interaction plays important role in overall magnetic properties as well.
\end{abstract}

PACS: 82.80.Ej, 81.07.-b, 75.75.-c, 81.16.-c

\section{Introduction}

The magnetic nanoparticles have been under investigation for a decades. The ferrite nanoparticles are important for biological applications. Many different modifications of the synthesis procedure are studied to improve the desired (for application) properties of the system $[1,2]$. The interesting modification is the construction of the core-shell particles produced by the step-bystep synthesis. Each step can lead to a different composition of the layer and in this way the particles can posses quite exotic structures with fine tunable properties. Each surface modification causes changes of magnetic properties nanoparticles [3-5].

\section{Experimental}

The ferrite nanoparticles were made according to the modified procedure developed originally by S. Sun [6]. The main idea of usage the $\mathrm{Fe}(\mathrm{acac})_{3}$ as a precursor for high-temperature decomposition reaction in presence of phenyl ether, alcohol, oleic acid and oleylamine was taken from publication [6]. We have adapted the idea how to grow bigger core-shell particles in gradual manner. In the first step the seeds particles were made from $1 \mathrm{mmol}$ solution of $\mathrm{Fe}(\mathrm{acac})_{3}$ complex decomposed at $260^{\circ} \mathrm{C}$ and obtained fluid was divided into 4 even parts. Next, the following growth on seeds was made with the concentration of $\mathrm{Fe}(\mathrm{acac})_{3}$ complex or both $\mathrm{Fe}(\mathrm{acac})_{3}$ and $\mathrm{Fe}(\mathrm{acac})_{2}$ complexes ranged from 1 to $8 \mathrm{mmol}$ [7].

The room temperature Mössbauer spectra was taken with the spectrometer working in constant acceleration mode with Co in Rh matrix as a source.

\section{Results and discussion}

In Fig. 1 the TEM images of selected samples used in article which have been produced from $\mathrm{Fe}(\mathrm{acac})_{3}$ complex or both $\mathrm{Fe}(\mathrm{acac})_{2}$ and $\mathrm{Fe}(\mathrm{acac})_{2}$ complexes are depicted, Figs. 1a-b and Figs. 1c-d, respectively.
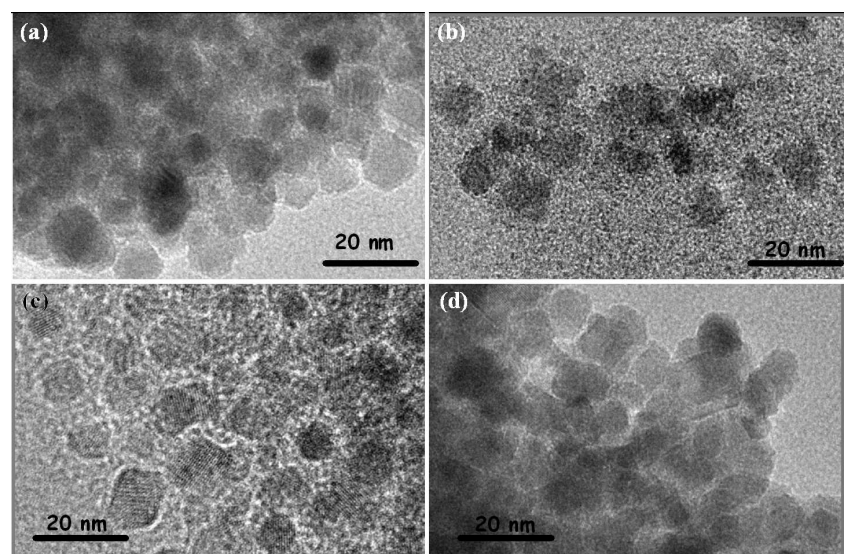

Fig. 1. The TEM images of: $(\mathrm{a}-\mathrm{b})$ the maghemite particles obtained from $2 \mathrm{mmol}$ and $6 \mathrm{mmol} \mathrm{Fe}(\mathrm{acac})_{3}$ added in the second step of procedure, respectively; (cd) magnetite nanoparticles $2 \mathrm{mmol}$ and $8 \mathrm{mmol}$ both $\mathrm{Fe}(\mathrm{acac})_{3}$ and $\mathrm{Fe}(\mathrm{acac})_{2}$ added in the second step of procedure, respectively.

The analysis of the images shows that the average size and size distribution are of the same order in all presented samples. The average particles size is ranging from $9.0 \pm 0.5$ to $10.0 \pm 0.5 \mathrm{~nm}$ regardless of the amount of precursor taken. It is seen from Fig. 1 that the maghemite nanoparticles in case of $6 \mathrm{mmol}$ procedure are more separated if compared to $2 \mathrm{mmol}$ one. A opposite trend is observed for magnetite nanoparticles. The nanoparticles made in the procedure with $2 \mathrm{mmol}$ are more separated than $8 \mathrm{mmol}$ one.

In the Fig. 2 the room temperature Mössbauer spectra of four series of the samples are collected. In panel (a) are presented the spectra where the only $\mathrm{Fe}(\mathrm{acac})_{3}$ com- 
plex was used in both steps of the main synthesis procedure and finally pure maghemite particles were obtained. In panel (b) the Mössbauer spectra are collected of the particles obtained from the mixture of $\mathrm{Fe}(\mathrm{acac})_{3}$ and $\mathrm{Fe}(\mathrm{acac})_{2}$ in the stechiometric ratio $2: 1$ what should lead to pure magnetite. In panels (c) and (d) the spectra of core-shell type of the particles are shown: magnetite on maghemite and maghemite on magnetite, respectively.

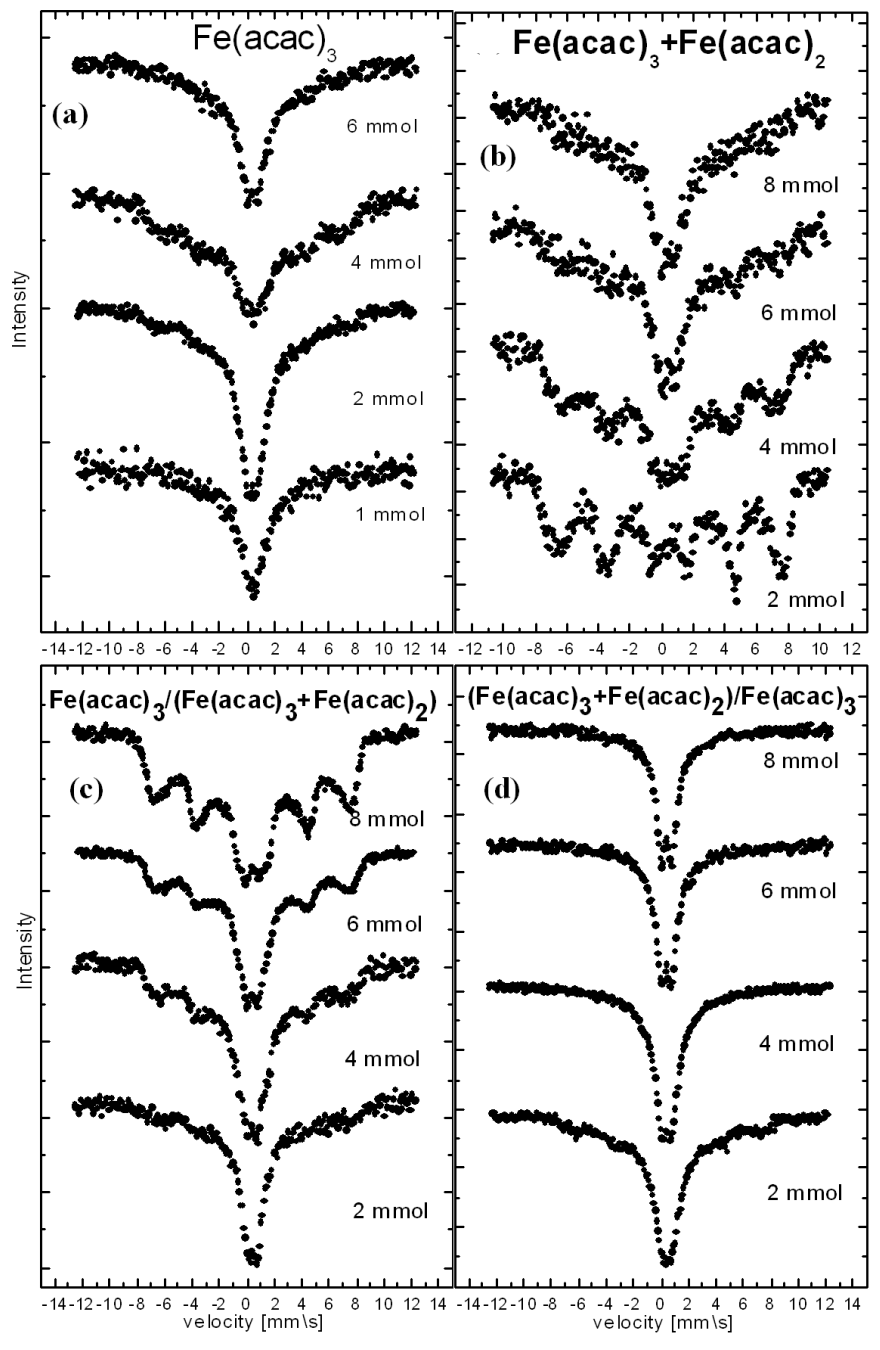

Fig. 2. The Mössbauer spectra at: monophase maghemite (a) and magnetite (b) nanoparticles; and of core-shell nanoparticles with different composition in each layer: (c) magnetite on maghemite, (d) maghemite on magnetite.

In Fig. 2a four Mössbauer spectra of the maghemite nanoparticles are plotted. These samples are assigned as $1 \mathrm{mmol}, 2 \mathrm{mmol} 4 \mathrm{mmol}$ and $6 \mathrm{mmol}$, which means how much of $\mathrm{Fe}(\mathrm{acac})_{3}$ complex was used in the second step of the procedure. The obtained spectra for mentioned set of the particles have following features. For the smallest concentration of iron acetate complex the spectrum consists of relatively broad singlet. It shows typical superparamagnetic behaviour for interacting par- ticles at room temperature which agree with published results [8]. All nanoparticles in the sample (1 mmol) have relaxation times larger than the time scale of Mössbauer spectroscopy (i.e. $>5 \times 10^{-9} \mathrm{~s}$ ). For largest iron acetate concentration the spectrum is superposition of two components the quadrupole doublet in the middle and broad singlet.

In the Fig. $2 \mathrm{~b}$ four samples of pure magnetite were tested with different concentration of constituents taken in second step of the synthesis. Here the total amount of $\mathrm{Fe}(\mathrm{acac})_{3}$ and $\mathrm{Fe}(\mathrm{acac})_{2}$ is varied between 2 and $8 \mathrm{mmol}$ in steps of $2 \mathrm{mmol}$. The relative molar ratio between $\mathrm{Fe}^{3+}$ and $\mathrm{Fe}^{2+}$ compounds were kept 2:1 as is typical for stoichiometric magnetite. The astonishing fact is that for lower complex concentration the spectra are magnetically splitted and with increase of the concentration the gradual transition to the superparamagnetic state is observed. It can be concluded that the blocking temperature is lower for higher complex concentration.

In Figs. 2c and 2d the effect of next step of the modification is shown. The two series of the four samples are depicted. In these two cases the particles are build as a core-shell type of the structure where two layers posses different chemical composition. The aim was to obtain magnetite on maghemite and vice versa.

Figure 2c present magnetite on maghemite particles where layer-shell built up from mixed $\mathrm{Fe}(\mathrm{acac})_{3}$ and $\mathrm{Fe}(\mathrm{acac})_{2}$ complexes is deposited on pure $\mathrm{Fe}(\mathrm{acac})_{3}-$ core. It can be seen here that the increase of $\left(\mathrm{Fe}(\mathrm{acac})_{3}\right.$ and $\mathrm{Fe}(\mathrm{acac})_{2}$ ) complexes concentration results in more magnetically split spectra. The quadrupole doublet in the central part of the spectra is however present all the time and due to nanoparticles with the relaxation time shorter than the time scale of Mössbauer spectroscopy. The intensity of splitted part of the spectra increases with increase of complex concentration. Such scenario has been observed for pure interacting maghemite particles (see above).

The Mössbauer spectra for core-shell (maghemite on magnetite) particles are collected in Fig. 2d. Here the main part of the spectra is the quadrupole doublet and the broad single line for which the broadening decreases with increase of complexes concentration. Thus the relaxation time increases with shell thickness.

At the first glance can be seen that the interacting particles with the composition typical for maghemite are in superparamagnetic state at room temperature. The spectra in serie possess the shape which suggests continuous transformation from superparamagnetic to magnetic state with blocking temperature above room temperature.

The spectra obtained for particles which were synthesized with stoichiometric composition of $\mathrm{Fe}$ ions as in magnetite are close superparamagnetic blocking temperature. Two spectra with lowest complex concentration have quite well resolved sextets typical for magnetite. Two other are slightly below blocking temperature and superparamagnetic part dominates the spectra. 
For core-shell particles the qualitative changes of the Mössbauer spectra are very complex. First, the reason for seeing superparamagnetic state is dominated by shell layer. Pure maghemite (Fig. 2a) was rather superparamagnetic at room temperature for all concentrations. Set of the core-shell samples (Fig. 2b) with maghemite at the surface shows also mostly superparamagnetic behavior. In the series with magnetite in the shell layer some of the samples blocking temperature is below room temperature. However, the changes of the spectra with concentration of used complex during shell layer deposition strongly depends on the core composition and are of the same character as were seen for monophase particles. The magnetic splitting increases with concentration for maghemite and decreases for magnetite.

The observation is somewhat unambiguous but it has only proven once more that the explanation of nanoparticles behaviour in not an easy task. The intraparticle magnetic interaction plays important role in the system as well. It should be considered strong interaction between separate layers inside the particles. The surface effects also differently influences the stechiometric magnetite and maghemite what can be seen as a difference between Mössbauer spectra for these two compositions. In such instance more investigations of the problem must be done in the future.

\section{Conclusions}

1. The complex concentration has rather minor influence on particle size.

2. The magnetic properties on the particles are strongly modified by complex stoichiometry.

3. The core-shell particles show importance of intraparticle interaction.

4. Core govern the changes with concentration, while shell layer is responsible for superparamagnetic blocking temperature.

\section{Acknowledgement}

Work partly supported by Polish National Grant No. N N204 246435.

\section{References}

[1] F. Sulek, M. Drofenik, M. Babulin, Z. Knez, J. Magn. Magn. Mater. 322, 179 (2010).

[2] D. Ortega, J.S. Garitaonandia, C. Barrera-Solano, M. Ramirez-del-Solar, E. Blanco, M. Dominguez, J. Non-Cryst. Solids 352, 2801 (2006).

[3] E. Tronc, A. Ezzir, R. Cherkaoui, C. Chanehac, M. Nagues, H. Kachkachi, D. Fiorani, A.M. Testa, J.M. Greneche, J.P. Jolivet, J. Magn. Magn. Mater. 221, 63 (2000).

[4] A. Millan, A. Urtizberea, N.J.O. Silva, F. Palacio, V.S. Amaral, E. Snoeck, V. Serin, J. Magn. Magn. Mater. 312, L5 (2007).

[5] H. Kachkachil, A. Ezzir, M. Nogu, E. Tronc, Eur. Phys. J. B 14, 681 (2000).

[6] S. Sun, H. Zeng, J. Am. Chem. Soc. 124, 8204 (2002).

[7] B. Kalska, will be published.

[8] J. Tucek, R. Zboril, Czech. J. Phys. 55, 7 (2005); S. Morup, E. Tronc, Phys. Rev. Lett. 72, 3278 (1994); L.T. Kuhl, K. Lefmann, C.R.H. Bahl, S.N. Ancona, P.-A. Lingrad, C. Frandsen, D.E. Madsen, S. Morup, Phys. Rev. B 74, 184406 (2006); T. Belin, N. GuigueMillot, T. Caillot, D. Aymes, J.C. Niepce, J. Solid State Chem. 163, 459 (2002); D. Fiorani, A.M. Testa, F. Lucari, F. D'Orazio, H. Romero, Physica B 320, 122 (2002). 